\title{
El asociativismo en las empresas de diseño de indumentaria de autor en Argentina
}

Sofía Marré *

\begin{abstract}
Resumen: El asociativismo como alternativa de sustentabilidad económica en las micro, pequeñas y medianas empresas de diseño de indumentaria de autor. Las ventajas de trabajar de manera conjunta se traducen en la representación institucional, la capacidad de negociación, mejorar la comercialización, aumentar la difusión y comunicación, compartir personal calificado, incorporar tecnología que es inaccesible a nivel individual, intercambiar experiencia y saberes entre colegas, tener mayor objetividad al momento de identificar problemas y sus posibles soluciones, acceder a capacitaciones, mejorar las oportunidades y tener mejor acceso a líneas de financiamiento.
\end{abstract}

Palabras claves: aglutinar - asociativismo - colaborar - creatividad - equipo - indumentaria de autor - potenciar - sustentabilidad - unificar esfuerzos.

[Resúmenes en inglés y portugués en la página 69]

${ }^{(*)}$ Formada en el ámbito de las organizaciones productivas. En el 2009, cursó el Posgrado internacional de Gestión y Política en Cultura y Comunicación en la Facultad Latinoamericana de Ciencias Sociales (FLACSO). En el 2006 fue becada por la Universidad de San Andrés, la Universidad Torcuato Di Tella y Cedes, en el Posgrado de Managment de Organizaciones son fines de lucro. Actualmente se desempeña como Coordinadora de la Oficina de Moda del Centro Metropolitano de Diseño. [Ver CV completo en página 206]

El diseñador de autor diseña con identidad, produciendo bienes diferenciados que responden a la lógica de consumo de una sociedad que, desde los 90, comenzó a perfilarse más individualista, atreviéndose a buscar su propio estilo en el vestir. Esta demanda nació de la búsqueda por diferenciarse de las tendencias masivas, impuestas por grandes marcas extranjeras. El diseño de indumentaria de autor crea bienes con un fuerte carácter innovador y una fuerte impronta personal. Sus creadores experimentan con diversos procesos productivos y fusionan técnicas industriales con artesanales.

En nuestro país, el fenómeno del diseño de autor ha tenido un crecimiento sostenido desde el año 2001. Su alto valor agregado, y la diversidad de propuestas, han enriquecido la cultura local, llamando la atención de consumidores locales y foráneos. "Si la identidad es poder, resulta natural que la necesidad de generarla se convierta en una de las motivaciones más importantes que tienen los diseñadores” afirma la socióloga Susana Saulquin. 
(2008, p. 147). Las propuestas han surgido de la mano de diseñadores que optan por tener su propio emprendimiento y marca. En este sentido, esta industria posee bajas barreras de entrada por la limitada inversión económica, ya que la maquinaria y los insumos todavía son accesibles para quien esté pensando dedicarse al diseño de autor.

En la actualidad el sector está conformado por 232 empresas, de las cuales la gran mayoría, un $92 \%$, son microempresas, un $7 \%$ son pequeñas empresas y sólo un $1 \%$ son medianas empresas.

El reciente estudio elaborado por el INTI y la Fundación Pro Tejer indica que la facturación total anual de las empresas resulta ser de \$587 millones aproximadamente, y el empleo directo e indirecto se calcula en unas 3200 personas. La producción total de prendas se estima en 1.150 .000 unidades.

Geográficamente, la industria posee gran cobertura en el país, con empresas en 18 provincias. Sin embargo, la concentración es también muy alta, la Ciudad de Buenos Aires alberga el 38\% de las empresas del sector.

El 22\% de la totalidad de empresas posee más de 10 años en el Mercado y un $61 \%$ logró una permanencia de más de 4 años. Estos datos afirman que este tipo de emprendimientos tienen viabilidad en el tiempo. (INTI y Fundación Pro-Tejer, 2012)

En líneas generales, se trata de un sector que se ha formado, mayormente, en instituciones educativas relacionadas a carreras específicas del sector, como son las áreas de indumentaria y textil. Estas empresas enfrentan dificultades para llevar adelante una adecuada gestión porque las personas que las lideran en muchas casos no poseen formación o experiencia adecuada en gestión de desafíos críticos como promoción, comercialización, financiamiento, liderazgo de equipos, contabilidad, etc. En general se trata de firmas lideradas por un diseñador que cumple "de hecho" el rol de empresario.

Entre los desafíos y obstáculos comunes que estas empresas perciben para lograr la sustentabilidad en el tiempo está el incremento de costos, generado por una coyuntura económica inestable, con una inflación del 22\% anual, solo tomando el año 2012 de acuerdo a datos del IPC del INDEC para indumentaria e IPC 7 provincias. Las firmas no pueden trasladar todos los aumentos de costos a precios porque se convertirían en opciones muy caras para el mercado. (INDEC, 2012)

La insuficiente difusión de la marca o producto, se presenta en segundo lugar como una gran problemática. Se trata de un sector de riesgo, que apuesta a la diferenciación en sus propuestas con pequeñas estructuras comerciales para hacer frente al sistema de la moda, integrado por grandes centros que imponen tendencias mundiales de consumo.

En este sentido, el emprendedor debe buscar caminos alternativos a la alta inversión que requieren los espacios publicitarios en medios gráficos, televisivos y vía pública. El uso de redes sociales e Internet son los principales medios de difusión de estos emprendimientos, el $87 \%$ utiliza las redes sociales para difundir su marca y el $43 \%$ su página Web.

La falta de financiamiento, es otra problemática clave para el sector, el 64\% de las empresas se autofinancian reinvirtiendo ganancias del emprendimiento y aportando ahorros propios y un $14 \%$ establece planes de pago con sus proveedores. Sólo el 12\% logra obtener créditos, las empresas perciben intereses altos de parte de las entidades financieras y requisitos (garantías, documentación e inscripciones) que no están al alcance de su realidad. La escasez de personal calificado es un problema común a toda la industria de la moda 
local. Desde la crisis del 2001 y gracias a las políticas industriales, la industria textil retomó su actividad productiva luego de muchos años de estancamiento.

Y por último, la falta de conocimiento de canales comerciales, también es percibida como dificultad para la venta de estos productos diferenciados que requieren de una especial atención para insertarlos en el mercado. Analizar estrategias alternativas viables o factibles para este sector es sumamente necesario en un mercado cuya competencia es alta y demandante. El asociativismo pues, se presenta como una estrategia novedosa y una alternativa para las micro, pequeñas y medianas empresas de diseño de autor. La generación de alianzas, grupos asociativos, cooperativas son caminos para lograr un beneficio común al conjunto de participantes.

El asociativismo es considerado como un mecanismo de cooperación entre pequeños y medianos productores en donde cada productor participante, manteniendo su independencia jurídica y autonomía gerencial, decide voluntariamente participar en un esfuerzo conjunto con los otros participantes para la búsqueda de un objetivo común.

Las asociaciones o grupos asociativos surgen a partir de un conjunto de necesidades básicas que comparten las pequeñas y medianas empresas. El diseñar y organizar estrategias colectivas se presenta como una posibilidad de supervivencia. Además, en el trabajo en conjunto surgen nuevas posibilidades que deben traccionar de manera colectiva para obtener éxito.

Estos acuerdos entre empresas contribuyen a mejorar la competitividad. Se utilizan como un mecanismo frente a firmas de mayor poder de negociación con proveedores, canales comerciales y de promoción, entidades financieras, como forma de acceder a nuevos mercados y recursos, ganando en economías de escala y diversidad o para disminuir costos o riesgos. En la Argentina, las asociaciones entre empresas de diseño de indumentaria de autor son consideradas como un fenómeno reciente. Debido a que la mayoría comenzó sus actividades a partir del año 2001, son pocas las asociaciones de Pymes nucleadas entorno a esta posibilidad.

Analizaremos cuáles son las asociaciones que han surgido y que objetivos persiguen estas formas asociativas existentes que pueden servir de inspiración para pequeñas y medianas empresas que buscan soluciones a corto, mediano y largo plazo. Las estrategias utilizadas por estos grupos asociativos permiten a sus integrantes acceder a mayores niveles de rentabilidad, difusión y comercialización de sus productos.

\section{Grupo Palermo Viejo}

El Grupo Palermo Viejo es una asociación civil sin fines de lucro conformada en el año 2009 por un conjunto de marcas mayormente de indumentaria ubicadas en el barrio de Palermo Viejo. Esta asociación nació con el firme objetivo de posicionar la zona como un circuito de diseño creativo, innovador y de donde surgen importantes diseñadores, artistas y propuestas.

Desde su inicio el Grupo Palermo Viejo ha querido posicionarse como un barrio que respeta su idiosincrasia local, diferenciándose de otros que han querido otorgarle el nombre de Palermo Soho. 
Esta asociación nuclea “toda manifestación que tenga que ver con el diseño" explica Silvia Gil (comunicación personal, 2013), gerente de la asociación. El diseño se valora como factor diferencial de las propuestas masivas creadas por otras marcas. Entre las empresas que la conforman, hay adheridas tiendas de moda de cuidado posicionamiento, tiendas de diseño de indumentaria de autor, locales gastronómicos, hoteles boutique, estudios de fotografía y sofisticadas tiendas de decoración. La permanencia en el tiempo de locales de diseño de objetos, indumentaria, calzado, accesorios y otros, han hecho de Palermo Viejo un paseo al aire libre que propone una experiencia diferente a la de los shoppings.

El Grupo Palermo Viejo, tiene una representación institucional que es fundamental para acceder a diferentes organismos, tanto públicos como privados. El actuar de manera conjunta fortalece a la asociación y facilita el delineamiento de políticas acorde a las necesidades.

Han transcurrido tres años desde la iniciación de este grupo y muchas son las acciones que ha realizado. En primer lugar, difunde el circuito de locales comerciales organizando eventos para posicionar la zona a través de la generación de alianzas con diversas entidades. Esto ha resultado muy beneficioso como estrategia para posicionar el barrio. Los acuerdos con una entidad financiera han posibilitado otorgar descuentos y beneficios a los portadores de tarjetas de crédito en los locales participantes en jornadas con una alta difusión en prensa. Con las empresas privadas realizaron acciones concretas en determinadas fechas comerciales. El Gobierno local de la Ciudad de Buenos Aires también ha contribuido a difundir el barrio, junto al Centro Metropolitano de Diseño de la Ciudad de Buenos Aires, organizaron actividades e intervenciones en vidrieras con premios especiales.

En términos comerciales, lograron organizarse como una fuerza de venta alternativa que resolvió una necesidad puntual de una empresa grande que buscaba variedad de productos diferenciados. Esta alianza fue junto al Grupo Falabella, el retail de capitales chilenos. En junio de 2012, 8 marcas independientes pertenecientes al grupo desembarcaron en el local de Falabella del shopping Unicenter en un espacio exclusivo diseñado y ambientado con el espíritu del barrio de Palermo Viejo. "Esta acción fue sumamente enriquecedora, sumó para ambos lados, tanto las marcas como Falabella generaron nuevos públicos de consumidores, hubo mucha prensa" afirma Silvia Gil (comunicación personal, Enero 2013).

En efecto, fue tan exitosa la alianza, que el modelo luego se replicó en varias tiendas que Falabella tiene en Santiago de Chile y en Colombia. En esta oportunidad, no solo la difusión del barrio se hizo por primera vez en otro país, promocionando un circuito turístico alternativo de Buenos Aires, sino también las marcas participantes exportaron sus productos a través de la cadena, abriéndose a un nuevo mercado.

Los requisitos de compra de las grandes marcas de retail muchas veces imposibilitan a las emprendedores a cumplir en tiempo, forma y escala de producción. Esta acción demostró que es posible satisfacer la necesidad de gran volumen de compra de una cadena como Falabella ofreciendo como alternativa la suma de diferentes productos.

Como toda asociación civil, este grupo tiene un comité directivo que se reúne constantemente para analizar y decidir el rumbo y acciones a llevar a cabo. Los demás socios participan de un plenario que se realiza dos veces al año. En estas reuniones se muestran los resultados de acciones pasadas y una planificación de iniciativas a llevar adelante. 
La asociación tiene una estructura pequeña y muy efectiva. Silvia Gil es la gerente y encargada de coordinar a las empresas asociadas, elevar al comité directivo las propuestas y consultas, como así también ejecutar lo que allí se decide.

En la actualidad, el grupo tiene unas 100 marcas asociadas, que abonan una cuota mensual para solventar los gastos administrativos, honorarios, proveedores de servicios, difusión y comunicación del Grupo.

Entre los requisitos requeridos para participar de la asociación, Silvia sostiene "valoramos el compromiso, el respeto, el aporte y el entusiasmo de las empresas participantes, hay socios mas activos y otros mas pasivos como en toda asociación, lo importante es que todos apoyen las decisiones" (comunicación personal, Enero 2013).

En el camino transcurrido por la asociación surgieron algunos obstáculos que debieron sortear para seguir adelante, a modo de ejemplo, "la inscripción en IGJ demoró 2 años" dice Silvia. Pero claramente la balanza da positiva cuando de evaluar se trata "el intercambio entre diseñadores y grandes marcas ha resultado muy enriquecedor" agrega.

Al preguntarle a Silvia por los aprendizajes adquiridos en estos años afirma: "Los objetivos de una asociación deben ser concretos, claros, posibles pero sobre todo se necesita pasión para desarrollarlos, si no esta la pasión y el compromiso, la gente no te cree".

La visión, credibilidad y la dedicación de un grupo de empresarios del Circuito han sido fundamentales para aglutinar a las empresas "la credibilidad en este proyecto es dada por quienes lo lideran" dice Silvia.

\section{Proyecto Cuadrilla}

Proyecto Cuadrilla se formó en el año 2011, entre un grupo de emprendedores dedicados al diseño, producción y venta de prendas de diseño de indumentaria de autor. Los diseñadores que conforman esta novedosa propuesta son Catalina Ichaso y Lucinda Walmsley (Desastreria), Nicolás Bertolo (Urenko), Eduardo De Crisci (Decrisci), Diego Labrin Ladrón de Guevara (Ladrón de Guevara) y Fernando More (Fernando More). Egresados de la Universidad de Palermo y UBA en las carreras de diseño de indumentaria.

Proyecto Cuadrilla es una marca paraguas a la vez constituida por 5 marcas que tienen su propia identidad. Este grupo de diseñadores plantea una idea de integración y proyección en conjunto (www.decrisci.com/proyectocuadrilla/) Comparten una característica en común que es la de perseguir la originalidad y la innovación en sus productos, ya sea desde lo morfológico, experimentando con materiales convencionales y no convencionales, y desde la intervención de telas a través de diferentes técnicas de estampado, sublimados, engomados, entre otras.

Entre todos comparten saberes, alternativas, posibilidades y experiencias que colaboran en mejorar la performance de sus integrantes y ayudan al emprendedor a salir del aislamiento dado por sus propias preocupaciones y problemáticas cotidianas.

El trabajo interdisciplinario es fundamental, componen estructuras universitarias y artísticas diversas que enriquecen el alcance del discurso. "Cada uno posee una forma parti- 
cular de concebir la disciplina y el aprendizaje dando como resultado un paralelismo de constantes y variables, un intercambio dentro del propio hacer diseñador" dice De Crisci (comunicación personal, Enero 2013).

Así, el Proyecto Cuadrilla ha logrado tener más impacto que las propias marcas que lo componen de manera individual, potenciarse entre ellas les ha funcionado como alternativa para llamar la atención de los medios. A comienzos de 2012, fueron seleccionados por el Centro Metropolitano de Diseño para participar de una pasarela en el marco de BAFWEEK. La difusión obtenida en el desfile llamó la atención de medios gráficos que comenzaron a interesarse por estos jóvenes emprendedores.

En términos comerciales, el Proyecto Cuadrilla plantea una fuerza de venta en la cual las 5 marcas comparten una estructura edilicia, local a la calle, que se distingue del resto de sus competidores por su diversidad en la oferta. A mediados de 2012 se conformaron como una SRL e inauguraron su primer espacio de venta, estableciéndose físicamente en un local de Palermo. Este desafío los llevó a plantearse como seria el layout teniendo en cuenta cada marca, la administración, la contabilidad, la difusión, el stock y depósito, etc. Así, resolvieron que quien vende se lleva la ganancia y sólo se comparten gastos fijos y responsabilidades.

Los diseñadores comparten varias características que ellos mismos identifican, "somos jóvenes, pujantes, emergentes y con ganas de progresar en el ámbito de la moda" dice De Crisci. Los une el compromiso como valor de asociación, y agrega "no es algo que se remarque, esta implícito, somos todos dueños de igual manera, si yo perjudico al otro, nos perjudicamos todos." (comunicación personal, enero 2013)

Desde la asociación surgieron otros beneficios que ni ellos mismos se imaginaron al inicio: frente a la escasez de personal calificado resolvieron compartir talleres y mano de obra. De esta manera aseguraron una continuidad en la producción y generaron lealtad en sus proveedores de servicios, que reciben pedidos constantemente.

Además, se organizaron para generar la compra de materiales y telas en conjunto para obtener mejores precios. Al producir pequeños volúmenes, estos pequeños emprendimientos, no tienen una fuerza de compra para negociar con los proveedores mayoristas.

Para llevar a cabo las tareas cotidianas de un local, distribuyeron los roles de acuerdo a las capacidades de cada uno: el mantenimiento, el armado de gráfica, el desarrollo de propuestas comerciales, el contacto con proveedores, etc. "Desde el punto de vista personal son muchos los beneficios de esta unión, no solo por el posicionamiento en el mercado sino también porque estoy creciendo mucho profesionalmente, he aprendido mucho de la gestión empresarial y del aporte del otro" dice De Crisci (comunicación personal, Enero 2013). La agrupación tiene una mesa de diálogo constante en la que se discuten no solo aspectos de gestión empresarial, sino también aspectos productivos y proyectuales que hacen a la marca propia de cada uno. Encuentran dificultades como la falta de capital, mejorar la comunicación y lograr incrementar las ventas pero todos concuerdan que la experiencia en conjunto y la agrupación de personas dentro de un equipo es una buena vía para poder aunar fuerzas de apoyo y esfuerzos mutuos. 
El acceso al financiamiento, es una herramienta que esta asociación no ha utilizado aún, pero en donde la acción conjunta puede ser una alternativa para que estas empresas apliquen a créditos de forma más sencilla y trabajen con las entidades financieras para diseñar líneas de créditos acorde a las necesidades comunes.

\section{Ruta del Diseño, Salta}

La Ruta de Diseño en Salta se inició luego del 2011, año en el que el evento Por la Calle fue realizado en la ciudad de Salta organizado en conjunto por la Fundación Pro Tejer, el Observatorio de Tendencias de INTI Textiles y el Gobierno de la Ciudad de Salta.

El evento Por la Calle hizo foco sobre un fenómeno que ya estaba ocurriendo en la Ciudad, trazando y promocionando un circuito turístico entre los locales de venta de diseño de indumentaria de autor. Dicha acción sirvió de difusión sobre la presencia de diseñadores locales de Salta.

A principios de 2012, los mismos locales y diseñadores solicitaron a las organizaciones repetir este evento. Por falta de financiamiento no fue posible y esta situación dio lugar a la autogestión por parte de los propios locales que se organizaron para armar La ruta del Diseño. La propuesta, al igual que el evento Por la Calle, fue difundir el circuito de 8 locales de diseño de indumentaria.

Así, en septiembre de 2012, enmarcaron esta propuesta a la programación de la Semana de Moda de Salta organizada por el Gobierno local. Romina Zeitune una de las diseñadoras integrantes de este circuito y líder de esta iniciativa cuenta que "los 8 locales se pusieron de acuerdo para distribuir en un día folletos con mapas identificando los locales participantes y globos" (comunicación personal, febrero 2013).

Sin llegar a formar un grupo asociativo, lograron una acción conjunta que permitió difundir el circuito entre la prensa local, el turismo y el público en general. El liderazgo de Romina fue clave para llevar a cabo esta acción, "tenía tantas ganas de hacer este evento, que ejecuté yo misma varias de las tareas que teníamos que hacer, los 8 locales apoyaron las decisiones".

La experiencia fue exitosa, lograron ponerse de acuerdo entre los locales, llevar a cabo el evento y difundir el circuito. Ahora piensan replicarlo de igual manera este año para la misma fecha. "Para el próximo evento, hemos acordado distribuir mejor los roles y las tareas" agrega Romina. Construir y mantener la actividad de una asociación no es tarea fácil, requiere de dedicación y compromiso. Muchas veces, los miembros no están preparados para trabajar de manera asociativa, confunden roles, valores y responsabilidades. Es necesario dedicar tiempo a fortalecer las relaciones de grupo para lograr una asociación duradera capacitándose para trabajar de manera mancomunada, contar con herramientas que logren superar barreras u obstáculos, delinear y tener en claro los compromisos y obligaciones. 


\section{Sistemas Productivos Locales}

El Estado Nacional promueve la formación de grupos asociativos mediante el programa Sistemas productivos locales de la Sepyme del Ministerio de Industria de la Nación. Este programa brinda asistencia técnica y económica a asociaciones para implementar, desarrollar y/o fortalecer proyectos productivos que beneficien a todas las empresas participantes y a la comunidad a la que pertenecen.

Jazmín Castilla Sastre, Coordinadora del área de Diseño de la Dirección de Comercio del Gobierno de la Provincia de Salta, fue una de las precursoras en utilizar este programa alineando emprendimientos dedicados a la producción y venta de prendas de diseño de indumentaria de autor.

Entre 2009 y 2012 conformó dos grupos asociativos aglutinando 5 empresas de diseño respectivamente. En cada caso, aplicó para el Plan de Asociatividad para el Desarrollo Productivo, una de las cuatro líneas del programa de Sistemas Productivos Locales. Esta línea en particular, brinda asistencia técnica y económica a grupos asociativos nuevos o preexistentes en la ejecución de un plan de corto y mediano plazo con vistas a potenciar la competitividad de las empresas y el proceso de integración asociativa.

El programa dio la posibilidad de contratar un coordinador, "figura esencial para poder llevar a cabo el proyecto" según la Lic. Castilla Sastre (comunicación personal, enero de 2013). Este coordinador cumple la función de asistir al grupo en el cumplimiento del plan, así como en la formulación y el seguimiento del proyecto de inversión a presentar así como también garantiza un equilibrio entre las partes, lima asperezas y mejora los vínculos entre los miembros.

El coordinador es contratado por el plazo de un año en el cual el programa reintegra el $100 \%$ de sus honorarios durante los primeros 6 meses, y el 50\% durante los 6 meses restantes y aporta, mediante Aportes No Reembolsables (ANR), hasta el 60\% de un proyecto de inversión que beneficie al conjunto de empresas asociadas.

"La idea de conformar un grupo asociativo es posicionarse en el mercado como una cooperativa o asociación en la cual cada marca renueva su individualidad y se fortalece" afirma Castilla Sastre y agrega "se trabaja desde 3 aspectos fundamentales: el productivo, el comercial y en la capacitación de sus integrantes” (comunicación personal, Enero de 2013) Esta primera cooperativa de diseñadoras de indumentaria, calzado y accesorios fue denominada Diseñadores de Salta e integrada por Roxana Liendro (Santos Liendro), Virginia Torres (María Cruz), Lucrecia Moyano (Begonia), Alicia Lima (Tramas Andinas) y Miriam Mocci (El Acay).

Asociarse para adquirir nueva tecnología ha sido una alternativa para este grupo asociativo. El programa otorgó al primer grupo un subsidio de $\$ 120.000$ para la puesta en funcionamiento de un taller de uso común equipado con maquinaria nueva que permitió mejorar la calidad de su producción, experimentar nuevas técnicas, mejorar los tiempos de confección de las prendas y compartir saberes técnicos.

Una de las marcas asociadas utiliza la nueva maquinaria para dar soporte al resto de las marcas. Alicia Lima, quien realiza telas tejidos en telar, comenzó a proveer al resto de las integrantes de la cooperadora elaborando los zapatos, carteras y accesorios. Así una de ellas fabrica las telas y las demás integrantes las utilizan para elaborar los productos finales. 
Desde el punto de vista comercial, participaron bajo una misma marca en ferias organizadas por el Gobierno de Salta y compartieron un espacio de venta comercial para compartir gastos, hecho que posibilitó a las 5 marcas posicionarse en el mercado de manera diferenciada, ofreciendo un espacio de venta rico en oferta y variedad de productos. Además realizaron compras en conjunto de materiales e insumos para adquirir precios más accesibles. Esta primera experiencia dejó entrever que es necesario mantener una homogeneidad entre las edades de sus integrantes, "compartir un rango etario facilita la comprensión y la búsqueda de objetivos en común entre los integrantes”, asegura Castilla Sastre (comunicación personal, Enero 2013).

El segundo grupo fue conformado dos años más tarde, nuevamente con Castilla Sastre como coordinadora del equipo. En esta oportunidad Jazmín afirma "ya había adquirido experiencia de trabajo en equipo y saberes para perfeccionar mi accionar como coordinadora".

El grupo solicitó $\$ 250.000$ de aportes no reembolsables para la producción textil, calzado y accesorios. Esta segunda asociación está integrada por María Fernanda Perales (Mafe), Cecilia Santa Cruz (Cece), Virginia Rabaglia (Kikiriki); Gabriela Pernas (Tejido Bonito) y Débora Barni (Siento Volar).

Entre las principales dificultades que atraviesan estas diseñadoras para elaborar sus productos se destaca el tiempo que tardan en producir, una variable que les juega en contra para tener un volumen de mercadería óptimo para la venta y ofrecer a mayoristas. Conseguir la materia prima resulta costoso porque la mayoría de los materiales los compran en Buenos Aires.

Para tratar de incorporar nuevas materias primas, este grupo se propuso trabajar en $\mathrm{Se}$ clantás con la CUM (Comunidades Unidas de Molinos), una organización de segundo grado compuesta por 13 comunidades campesinas del Valle Calchaquí. El propósito de esta actividad es intercambiar técnicas con el grupo de tejedoras que ya elaboran tejidos con diseño y incorporar el fieltro y otras materias primas.

En el transcurso de este desafiante camino han sido muchos los aprendizajes adquiridos: trabajar de manera mancomunada, prepara mejor a este tipo de empresas a afrontar las problemáticas comunes y propias del sector. "Los diseñadores no están preparados para organizarse por si solos en un proceso asociativo, la figura del coordinador, que en este caso estuvo a mi cargo, fue clave para la continuidad y éxito de los objetivos" concluye Jazmín Castilla Sastre (comunicación personal, Enero 2013).

\section{Conclusiones}

Analizando cada uno de los grupos asociativos, concluimos en que el trabajo colectivo puede ser una estrategia saludable para las empresas. En este sentido, las asociaciones entorno a micro, medianas y grandes empresas de diseño de indumentaria de autor, ya comienzan a mostrar resultados interesantes.

Son muchas las ventajas de trabajar en esta modalidad: tener representación institucional, capacidad de negociación, mejorar la comercialización, aumentar la difusión y comunicación, compartir personal calificado, incorporar tecnología que es inaccesible a nivel 
individual, intercambiar experiencia y saberes entre colegas, tener mayor objetividad al momento de identificar problemas y sus posibles soluciones, acceder a capacitaciones, mejorar las oportunidades y tener acceso a financiamiento.

Habiendo analizado todos los casos entendemos que para lograr asociaciones duraderas y sustentables en el tiempo sus miembros deben tener claros los valores que hacen al trabajo en conjunto. Los valores son parte de un código de convivencia, que muchas veces se construyen mediante acuerdos tácitos y se basan en el compromiso, la responsabilidad, la cooperación, la equidad, la democracia y la solidaridad de sus integrantes.

En tanto, los miembros de una asociación deben también compartir valores éticos, como la honestidad, la transparencia, la responsabilidad social y la preocupación por los demás. Respetarlos y llevarlos a la práctica es un desafío para cada integrante, que en la búsqueda de lograr su propio beneficio debe observar si esta atentando al beneficio común.

Los principios de trabajo de estas asociaciones deben ser aprobados por el conjunto e implican participación voluntaria y abierta, el control democrático, la participación económica y la autonomía e independencia de los asociados.

Un asociativismo saludable debe superar el concepto de competencia entre los más pequeños y unir fuerzas para enfrentarse a los verdaderos competidores: los fuertes grupos económicos multinacionales con una creciente acumulación de capital y las grandes marcas que ya están establecidas en el mercado.

El liderazgo dentro de una organización asociativa debe ser aglutinador e inclusivo. Los líderes tienen el reto de cohesionar a personas con ideologías plurales. Deben ser personajes vitales y relevantes para las personas con las que colaboran y para los propósitos para los que trabajan. Asimismo deben ser creíbles por su experiencia y trayectoria.

Toda asociación debe trabajar en equipo, unificando esfuerzos de todos los miembros para cumplir los objetivos de manera de rápida, sencilla y eficiente. La coordinación y planificación de las acciones a llevar a cabo organizan la dinámica de participación de los integrantes. Desarrollar normas de convivencia y distribuir roles claros son puntos clave para que la asociación sea más efectiva. El consenso es importante para lograr una mayor participación y asegurar conformidad entre los miembros. Una buena comunicación interna y reuniones frecuentes colaboran en mejorar y fortalecer las relaciones entre los miembros. La búsqueda y adecuación de formas asociativas acordes a la realidad de las pequeñas y medianas empresas es materia que no se debe descuidar. Construir una asociación no es tarea fácil, siempre van a existir personas más activas que otras, lo importante es que a pesar de ello se puedan apoyar las decisiones, para que aquellos que las llevan a cabo puedan ejecutarlas satisfactoriamente. La unión hace la fuerza en la medida en que se respeten los derechos de los demás y se trabaje de manera mancomunada. 


\section{Referencias bibliográficas}

Bailey, E. (2012). El Trabajo Colectivo. Disponible en: http://white.oit.org.pe/spanish/ 260 ameri/oitreg/activid/proyectos/actrav/edob/material/pdf/archivo29.pdf

Bertolini, G. (2012). Fundación Cieso. Disponible en: http://www.fundacioncieso.org.ar/ testing-wp/wp-content/uploads/Los_emprendedores_asociativos_y_las_cadenas_productivas.pdf

Marino, P., Marré, S., Mon, L. (2012). Diseño de Indumentaria de Autor en Argentina 2012. Diagnóstico productivo e impacto económico basado en la Encuesta Nacional de Diseño de Indumentaria de Autor 2012, Buenos Aires: INTI y Fundación Pro Tejer

Proyecto Cuadrilla. (2011). Disponible en: http://www.decrisci.com/proyectocuadrilla/

Saulquin, S. (2008) ¿Por Qué Argentina? Buenos Aires: Ediciones del Paraíso.

INDEC (2012). Disponible en: CIFRA. Centro de Investigación y Formación de la Repú-

blica Argentina. http://www.centrocifra.org.ar

SEPYME.(2013). Secretaría Pyme y desarrollo regional. Disponible en: www.sepyme.gob.ar

Summary: The article approaches the associativism as an economic sustainability option in the micro, small and medium enterprises in clothing author design. The advantages of working together translate into institutional representation, negotiation skills, improve marketing strategies, increase communication, share skilled personnel, incorporate technology that is inaccessible to the individual level, to exchange experience and knowledge with colleagues, have greater objectivity in identifying problems and possible solutions, access training, improve opportunities and better access to credit lines.

Keywords: associativism - author clothing - collaborate - creativity - empower - gather efforts - sustainability - team - unite.

Resumo: $\mathrm{O}$ associativismo como alternativa de sustentabilidade econômica nas micro, pequenas e medianas empresas de design de indumentária de autor. As vantagens de trabalhar conjuntamente se traduzem na representação institucional, a capacidade de negociação, melhorar a comercialização, aumentar a difusão e comunicação, compartilhar pessoal qualificado, incorporar tecnologia que é inaccessível a nível individual, intercambiar experiências e saberes entre colegas, ter mais objetividade no momento de identificar problemas e suas possíveis soluções, aceder a capacitações, melhorar as oportunidades e ter melhor acesso a linhas de financiamento.

Palavras chave: aglutinar - associativismo - colaborar - criatividade - equipe - indumentária de autor - potenciar - sustentabilidade - unificar esforços. 